\title{
DESIGN OF INTEGRAL VARIABLE STRUCTURE CONTROL FOR NONLINEAR SYSTEM BASED ON CMAC NEURAL NETWORK AND REFERENCE MODEL
}

\author{
Lin Lei, Houjun Wang, Shuchun Zhou
}

School of Automation Engineering, University of Electronic Science and Technology of

China, Chengdu, Sichuan, China

Abstract: Because mismatch of models of uncertain nonlinear control system and external disturbance etc., these will make effects of controller designed badly in uncertain nonlinear system. In this paper, we put forward a method, which can improve performance of uncertain nonlinear control system. We divide nonlinear control system into reference model and uncertain parts two respectively design and last synthesize control law of two parts. We build up approximate reference model through information known, make use of integral variable structure control of exponential approach method, and adopt to integral variable structure control based on CMAC neural network for uncertain part. We make use of method of extension controller's design based on extension set theory while uncertain boundary unknown. The simulate experiment validate this control method can improve control quality in an extent.

Keywords: CMAC neural network; integral variable structure control; uncertain nonlinear system; robust control; reference model

\section{INTRODUCTION}

Because there are uncertainty and nonlinear property of system when design actual engineering controller, which makes design become difficult. We make use of method of Integral Variable Structure Control (IVSC) ${ }^{[1]}$, while we have known estimative parameter and uncertain boundary of system model; we may make use of method of extension controller's design ${ }^{[2]}$ based on extension set theory while uncertain boundary unknown. We 
both suppose that system model completely cannot be determined and design controller above two control method. In fact, sometimes we may make full use of information known to build up approximate system model. Although mismatch of model with actual system may be comparatively big, it can reflect some effective characteristic of actual system more or less. We call approximate system model to build up as reference model ${ }^{[3]}$. In this paper, we put forward integral variable structure control method of nonlinear system based on CMAC neural network and reference model. We confirm its validity through simulate and experiment.

\section{THE THEORY AND SYSTEM DESCRIPTION OF NONLINEAR CONTROL SYSTEM}

Define nonlinear system as: $x^{(n)}=f(\underline{x})+b u$

In formula (1),$\underline{x}=\left(x_{1}, x_{2} \ldots x_{n}\right)^{T}=\left(x, \dot{x}, \ddot{x}, \ldots x^{(n-l)}\right)^{T}$ is state vector, $f$ is continuous bounded function, $\mathrm{b}$ is unknown time-varying positive real even $0<b_{\min } \leq b \leq b_{\max }$, and $u \in R$ is input. Object of control is to supply an appropriate control input $u$ that it makes distance of error vector $\underline{e}=\underline{x}-\underline{x_{d}}=\left(e_{1}, e_{2}, \ldots ., e_{n}\right)^{T}=\left(e, \dot{e}, \ddot{e}, \ldots ., e^{(n-1)}\right)^{T}$ and zero vector become minimum. In this formula, $\frac{x_{d}}{=}\left(x_{d 1}, x_{d 2} \ldots x_{d n}\right)^{T}=\left(x_{d}, \dot{x}_{d}, \dot{x}_{d}, \ldots x_{d}{ }^{(n-1)}\right)^{T}$ is state vector of anticipant object.

Define reference model of nonlinear system built as follow:

$x_{m}^{(n)}=f_{m}\left(x_{m}\right)+b_{m} u_{m}$

Describe mismatch of model and actual system as follow:

$x_{\Delta}^{(n)}=f_{\Delta}\left(\underline{x_{\Delta}}\right)+b_{m} u_{\Delta}$
In above two formulas, $b_{m}$ is parameter estimate of $b$, let $b_{m}=\sqrt{b_{\min } \cdot b_{\max }}$, $\underline{x_{m}}$ is state variable of reference model, and $\underline{x_{\Delta}}$ is dispersion between state variable of reference model and state variable $\underline{x}$ of actual system. Also, suppose that the relations as follows are tenable.

$$
\begin{gathered}
\underline{x_{\Delta}}=\underline{x}-\underline{x_{m}} \\
f_{\Delta}\left(x_{\Delta}\right)=f(x)-f_{m}\left(x_{m}\right)
\end{gathered}
$$

In condition of suppose, nonlinear system may be divided into two respective parts: reference model as formula (2) and model mismatch as formula (3). We may use different control law $u_{m}$ and $u_{\Delta}$ for two parts to get optimal effect of control. 


\section{STRUCTURE OF CONTROL SYSTEM}

$\mathrm{P}$ When $f_{m}$ is known and $f_{\Delta}$ is uncertain, we may use approach method to resolve Integral Variable Structure Control (IVSC) $u_{m}$, use method of extension controller design $u_{\Lambda}$, and finally combine both as the ultimate control law. The control system structure is shown in figure 1[4]. The model controller $x_{d}$ makes state input $\frac{x_{m}}{-}$ of reference model trace anticipant state vector $\frac{x_{d}}{f}$. That is to make error vector $\underline{e_{m}}=\underline{x_{m}}-\underline{x_{d}}$ full approach to zero. If $f_{m}$ known, we may directly use exponential approach method to resolve IVSC $u_{m}$

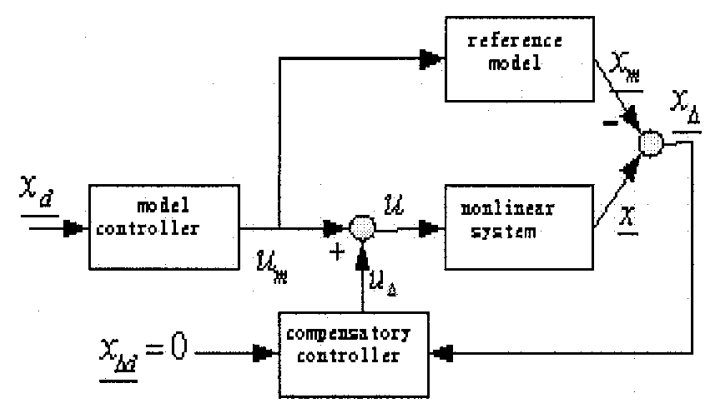

Figure 1. Structure of control system

\section{DESIGNING CONTROL LAW OF NONLINEAR CONTROL SYSTEM}

The switching function usually select linear function:

$$
S_{m}\left(e_{m}\right)=e_{m}^{(n-1)}+a_{1} e_{m}^{(n-2)}+\ldots . .+a_{n-1} e_{m}+K_{\mathrm{Im}} Z_{m}
$$

In above formula, define $Z_{m}=e_{m}$. Resolve derivative for (6) and get:

$S_{m}\left(e_{m}\right)=e_{m}^{(n)}+a_{1} e_{m}^{(n-1)}+\ldots . .+a_{n-1} e_{m}+K_{\mathrm{Im}} e_{m}$

Let $S_{m}$ and exponential approach law identical:

$S_{m}=-\varepsilon \operatorname{sgn}\left(S_{m}\right)-k S_{m}$

We may get $u_{m}$ from (7), (8) and (2) formula as follows:

$$
\begin{aligned}
& e_{m}^{n}+a_{1} e_{m}^{(n-1)}+\ldots . .+a_{n-1} \dot{e}_{m}+K_{\mathrm{Im}} e_{m}=-\varepsilon \operatorname{sgn}\left(S_{m}\right)-k S_{m} \\
& x_{m}^{(n)}-x_{d}^{(n)}+a_{1} e_{m}^{(n-1)}+\ldots . .+a_{n-1} \dot{e}_{m}+K_{\mathrm{Im}} e_{m}=-\varepsilon \operatorname{sgn}\left(S_{m}\right)-k S_{m} \\
& f_{m} \frac{\left(x_{m}\right)+b_{m} u_{m}-x_{d}^{(n)}+a_{1} e_{m}^{(n-1)}+\ldots . .+a_{n-1} \dot{e}_{m}}{+} K_{\mathrm{Im}} e_{m}=-\varepsilon \operatorname{sgn}\left(S_{m}\right)-k S_{m}
\end{aligned}
$$

Get from a a bove formula as follows:

$$
u_{m}=b_{m}^{-1}\left\{u_{m}-f_{m}\left(x_{m}\right)-\varepsilon \operatorname{sgn}\left(S_{m}\right)-k S_{m}\right\}
$$




$$
\hat{u}_{m}=x_{d}^{(n)}-a_{1} e_{m}^{(n-1)}-\ldots . .-a_{n-1} \dot{e}_{m}-K_{\mathrm{Im}} e_{m}
$$

Designing compensatory controller may adopt method to design extension controller [2]. The designing object of control $x_{m}^{\text {law }} u_{\Delta}$ is to compensate for dispersion between model's state vector $\frac{x_{m}}{x}$ and actual system' $\bar{x}$ state vector $\underline{x}$, which is to make $\frac{x_{\Delta}}{x}$ full closer to anticipant value $\underline{x_{d d}}$. According to Integral Variable Structure principle, define switch surface as follow:

$$
S\left(\underline{e_{\Delta}}\right)=e_{\Delta}^{(n-1)}+a_{1} e_{\Delta}^{(n-2)}+\ldots . .+a_{n-1} e_{\Delta}+K_{I} Z_{\Delta}
$$

$\underline{e_{\Delta}}=\underline{x_{\Delta}}-\underline{x_{\Delta d}}, \dot{Z}_{\Delta}=e_{\Delta}$ in the above formula. The control input $u_{\Delta}$ designed must satisfy sliding condition: $S S \leq-\eta|S|$

In the above formula, $\eta$ is a positive definite constant. We may design control law $u_{\Delta}$ of compensatory controller from invariable structure control law. Define Lyapunov function as $V=0.5 S^{2}$. Thus

$$
\dot{V}=S \dot{S}
$$

$=S \cdot\left(e_{\Delta}^{(n)}+a_{1} e_{\Delta}^{(n-1)}+\ldots . .+a_{n-1} \dot{e_{\Delta}}+K_{I} e_{\Delta}\right)$

$=S \cdot\left\{f_{\Delta}\left(x_{\Delta}\right)+b u_{\Delta}-x_{\Delta d}^{(n)}+a_{1} e_{\Delta}^{(n-1)}+\ldots . .+a_{n-1} \dot{e}_{\Delta}+K_{I} e_{\Delta}\right\}$

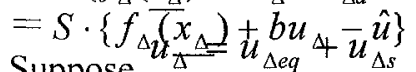

Suppose $u_{\triangle} u_{\Delta e q}+u_{\Delta s}$. In formula (14), ${ }^{u_{\Delta e q}}$ is equivalent control and $u_{\Delta s}$ is striking control. While $V=0$, we may get a optimal equivalent control $u_{\Delta e q}$ :

$$
u_{\Delta e q}^{*}=b^{-1}\left(\hat{u}-f_{\Delta}\left(\underline{x_{\Delta}}\right)\right)
$$

Because $b$ is unknown, we use $b_{m}$ as an approximate of $b$ and get an equal control $u_{\Lambda e q}$ :

$$
u_{\Delta e q}=b_{m}^{-1}\left(\hat{u}-f_{\Delta}\left(\underline{x_{\Delta}}\right)\right)
$$

Thereinto, $\hat{u}=-\left(a_{1} e_{\Delta}^{(n-1)}+a_{2} e_{\Delta}^{(n-2)}+\ldots . .+a_{(n-1)} \dot{e}_{\Delta}+K_{I} e_{\Delta}\right)$

Determinate striking control $u_{\Delta s}$ through Lyapunov theory and method as follows:.

$$
\begin{aligned}
& V=S S \\
& =S \cdot\left\{f_{\Delta}\left(\underline{x_{\Delta}}\right)+b u_{\Delta}-\hat{u}\right\} \\
& =S \cdot\left\{f_{\Delta}\left(\overline{x_{\Delta}}\right)+b u_{\Delta e q}+b u_{\Delta s}-\hat{u}\right\} \\
& =S \cdot\left\{f_{\Delta}\left(\overline{x_{\Delta}}\right)+\left(b b_{m}^{-1}-1\right) \hat{u}-b b_{m}^{-1} f_{\Delta}\left(\underline{x_{\Delta}}\right)+b u_{\Delta s}\right\} \\
& \quad u_{\Delta s}=\overline{b_{m}^{-1}}\left(f_{\Delta}\left(\underline{x_{\Delta}}\right)-G^{*} \operatorname{sgn}(S)\right) \text { and expression of } G^{*} \text { is as follows: }
\end{aligned}
$$$$
G^{*}=b_{m} b^{-1} \eta+\left|b_{m} b^{-1} f_{\Delta}\left(\underline{x_{\Delta}}\right)+\left(1-b_{m} b^{-1}\right) \hat{u}\right|
$$ 
We may confirm $\dot{V}=S \dot{S} \leqslant-\eta|S| \leq 0$ if we induce $u_{\Delta s}$ and $G^{*}$ into the formula $\dot{V}=S \dot{S}$.

We find that $u_{\Delta s}=b_{m}^{-1}\left(f_{\Delta}\left(x_{\Delta}\right)-G^{*} \operatorname{sgn}(S)\right)$ can satisfy sliding condition. So synthesis control law from equivalent control and striking control is as follows:

$$
\begin{aligned}
u_{\Delta} & =u_{\Delta e q}+u_{\Delta s} \\
& =b_{m}^{-1}\left(\hat{u}-f_{\Delta}\left(\underline{x_{\Delta}}\right)\right)+b_{m}^{-1}\left(f_{\Delta}\left(x_{\Delta}\right)-G^{*} \operatorname{sgn}(S)\right) \\
& =b_{m}^{-1}\left(\hat{u}-G^{*} \operatorname{sgn}(S)\right)
\end{aligned}
$$

Because $b$ is unknown and $f_{\Delta}$ is completely uncertain, $G^{*}$ cannot implement. We use CMAC neural network and make it full approach $G^{*}$ through study and training.

Suppose that input of CMAC network is as follows [5]: $G_{N}\left(\underline{e_{\Delta}} \mid \underline{w}\right)=\underline{w}^{T} \underline{\mu}\left(\underline{e_{\Delta}}\right)$. In above formula, $\underline{w}$ is a weighted vector and $\left.\underline{\mu\left(e_{\Delta}\right.}\right) \in R^{n \times 1}$ is a basis function of network. Suppose there is an optimal weight value $\underline{w}^{*}$ that makes $\varepsilon=G_{N}\left(e_{\Delta} \mid \underline{w}^{*}\right)-G^{*}$ minimum. A renewed law of weighted vector may be determined by Lyapunov theory for guaranteeing stability of system.

Suppose Lyapunov function as $V=0.5\left(S^{2}+b b^{-1} \lambda^{-1} \underline{\xi^{T}} \underline{\xi}\right), \underline{\xi}=\underline{w}-\underline{w}^{*}$.

$$
\begin{aligned}
& \dot{V}=S \dot{S}+b b^{-1} \lambda^{-1} \underline{\xi} \underline{\xi} \\
& =S \cdot\left\{f_{\Delta}\left(\underline{x_{\Delta}}\right)+\left(b b_{m}^{-1}-1\right) \hat{u}-b b_{m}^{-1}\left(G^{*} \operatorname{sgn}()\right)\right\}+b b_{m}^{-1}|S| \varepsilon \\
& \left.+\underline{\xi^{T}}|S| \underline{\mu}\left(\underline{e_{\Delta}}\right)-\lambda^{-1} \underline{\xi}^{T} \underline{\dot{w}}\right) \\
& \leq-\eta|S|-b b_{m}^{-1}\left(|S| \varepsilon+\underline{\xi^{\prime}}|S| \underline{\mu}\left(\underline{e_{\Delta}}\right)-\lambda^{-1} \underline{\xi^{T}} \underline{\dot{w}}\right) \\
& =-|S| \cdot\left\{\eta+b b_{m}^{-1} \varepsilon\right\}+b b_{m}^{-1} \underline{\xi^{T}}\left\{-|S| \underline{\mu}\left(\underline{e_{\Delta}}\right)+\underline{\dot{w}} \lambda^{-1}\right\} \\
& \dot{\underline{w}}=\lambda|S| \underline{\mu}\left(\underline{e_{\Delta}}\right) \text { is tenable, we may get that an inequality (19) is tenable. } \\
& \dot{V} \leq-|S|\left\{\eta+b b_{m}^{-1} \varepsilon\right\}
\end{aligned}
$$

Only if $\varepsilon$ is full closer zero, we must get $\eta+b b_{m}^{-1} \varepsilon \geq 0$, thus $\dot{V} \leq 0$. According to above deduction, choose study equation of CMAC network as follow:$$
\underline{\dot{w}}=\lambda|S| \underline{\mu}\left(\underline{e_{\Delta}}\right)
$$ 
According to literature ${ }^{[2]}$ discussion, we divide movement space of system into eligible region and ineligible region by size of $S$ and adopt different control in the different regions. Furthermore, we transfer sign function $\operatorname{sgn}(\cdot)$ into saturation function sat $(\cdot)$ for reducing phenomenon of vibration. Thus, the control law of compensatory controller may modify as

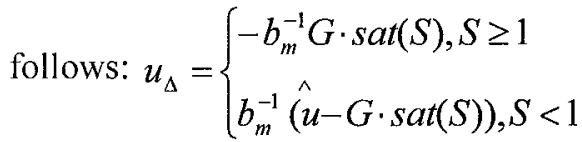

We sum formula (9) and formula (21), get a final control law of system as follows: $u=u_{m}+u_{\Delta}$

\section{SIMULATION AND DISCUSSION}

Confirming actual example is to use nonlinear system equation as follows: $\ddot{x}+(0.5+0.2 \sin (t)) \dot{x}+3.2 \cos (x)=(1+0.2 \sin (t)) u$

Suppose that reference model built is as follows:

$$
\ddot{x}+0.5 \dot{x}+3 \cos (x-0.5)=b_{m} u
$$

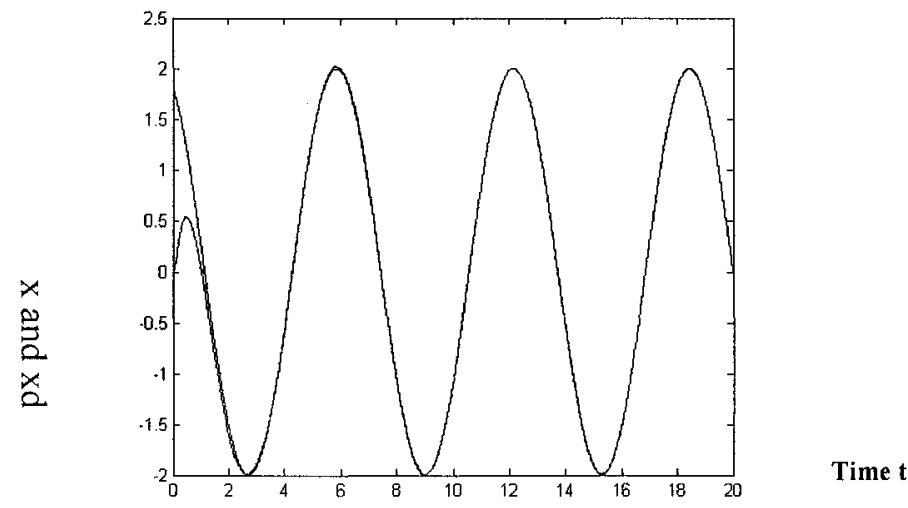

Figure2 output response of state vector

Evidently, $b=1+0.2 \sin (t)$, estimative value of $\mathrm{b}$ is $b_{m}=0.98$. Suppose that the anticipant state of system or instruction input is as follows: $x_{d}=2 \sin (t+2), a_{1}=1, a_{2}=2$. In the formula (8), let $\varepsilon=1, k=10$. A simulative result is shown in figure 2 and figure 3 . We find through simulation that inducing the project can more improve system performance of dynamic state and static state, make system steady, make system strong adaptability, make parameter definite easier, make effect of each parameter little, not demand more precise for parameter in arithmetic, make one-off determination. Also it confirms that controllable method in the paper has a good performance of track. 


\section{CONCLUSIONS}

In this paper, we put forward an designing method of uncertain nonlinear systemic controller that can eliminate phenomenon of integral saturation, decrease overshoots, make system adaptability strong and steady, make parameter definite easier, make effect of each parameter little, make one-off determination for parameter in arithmetic. Also we find through simulation that inducing the project can more improve sy

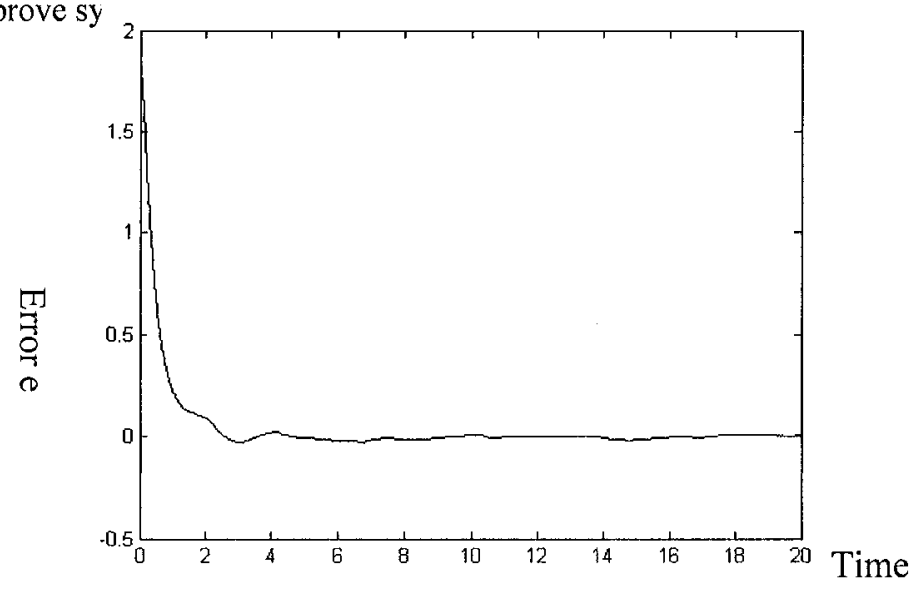

Figure 3 the error curve of reference model

\section{REFERENCES}

[1] Chin-Pao Hung. Integral Variable Structure Control of Nonlinear System Using a CMAC Neural Network Learning Approach. IEEE Trans. Syst., Man, Cybern.B, 2004, vol.34, no.1, pp. 702 709

[2] Chen Jenyang, Wong Chingchang. Extension Controller Design via Sliding Mode Control. Engineering Science, Sep. 2001, Vol.3, No.9, PP: 48 51

[3] Li Xinzhong, Jian Linke, Heyue. Neural Network Control for Nonlinear System Model Reference. Information and Control. Vol.25, No.6, 367 372, 1996

[4] David E, Kwon S. Neighborhood Sequential and Random Training Techniques for CMAC. IEEE Tran. Neural Networks, 1995, 6(1): 196 202.

[5] King-Lung Huang etc. Cascade-CMAC Neural Network Applications on the Color Scanner to Printer Calibration. Proceedings of the 1997 IEEE International Conference on Neural Networks, 1997.10 15 